\title{
Intra-car Wireless Sensors Data Collection: A Multi-hop Approach
}

\author{
Morteza Hashemi*, Wei Si*, Moshe Laifenfeld ${ }^{\dagger}$, David Starobinski* and Ari Trachtenberg* ${ }^{*}$ Dept. of \\ Electrical and Computer Engineering, Boston University, USA \\ Email: mhashemi@bu.edu,weisi@bu.edu, staro@bu.edu, trachten@bu.edu †Wireless Enablers Group, \\ GM Advanced Technical Center, Israel \\ Email: moshe.laifenfeld@gm.com
}

A version of this paper appeared as:

- Morteza Hashemi, Wei Si, Moshe Laifenfeld, David Starobinski, and Ari Trachtenberg, "Intra-car Wireless Sensors Data Collection: A Multi-hop approach," IEEE Vehicular Technology Conference, Dresden, Germany, June 2-5, 2013.

\begin{abstract}
We experimentally investigate the benefits of multi-hop networking for intra-car data aggregation under the current state-of-the-art Collection Tree Protocol (CTP). We show how this protocol actively adjusts collection routes according to channel dynamics in various practical car environments, resulting in performance gains over single-hop aggregation. Throughout our experiments, we target traditional performance metrics such as delivery rate, number of transmissions per packet, and delay, and our results confirm, both qualitatively and quantitatively, that multi-hop communication can provide a reliable and robust approach for data collection within a car.
\end{abstract}

\section{Index Terms}

wireless sensor networks, multi-hop networking, data collection, intra-car environments

\section{INTRODUCTION}

Recently, wireless sensor networks (WSN) have been considered for intra-vehicle wireless communications of sensors, switches and actuators. WSN have the potential to reduce cost, weight and maintenance of in-vehicle communications over wires in a scalable fashion. However, the ability to reliably aggregate data in one or several processing centers is critical to the monitoring capabilities of these WSN, as the sensors themselves are typically constrained in both energy and computational power. In vehicular environments, this aggregation is complicated by the dynamic channel properties that the car experiences as it travels through areas with different radio interference patterns, such as urban versus highway driving, or even different road quality, which may physically perturb the sensors.

To date single-hop communication, based on Zigbee, RFID, and ultra-wideband technologies ([1]-[2]), have been examined for replacing the current wires with wireless links. The general model in these works involves sensors and a central node (collection root), which are arranged in a star topology where different nodes communicate in a point- to-point fashion with the central node, without any networking protocol deployed.

In this paper we aim to explore the use of multi-hop communication within real vehicular environments under practical scenarios. Though there exist a number of data collection protocols for WSN in the literature, we have chosen to investigate the Collection Tree Protocol (CTP) [3] because it is widely deployed and well researched. We compare performance of CTP (which we associate with multi-hop communication throughout this paper) to a star topology protocol prevalent in the literature. Our experiments cover static and dynamic in-vehicle conditions, such as when the car engine is on, as passengers move in and out of the car, and when there exists external WiFi interference. Our results verify that multi-hop routing is functional inside the vehicle, and that it can achieve more reliable and robust performance compared with a star protocol.

The rest of the paper is organized as follows. Section II presents related work on intra-car WSN experiments, and background on CTP's working mechanism. Our experimental setup and methodology is described in Section III, 
and we compare CTP performance with a star topology protocol in Section IV. Section V demonstrates the adaptive behavior of CTP under dynamic conditions, and Section VI concludes the paper.

\section{BACKGROUND}

\section{A. Related work}

Several intra-car WSN experiments have been conducted for $915 \mathrm{MHz}$ and $2.4 \mathrm{GHz}$ ISM band. The authors in [1] report physical layer characterizations, e.g., Received Signal Strength Indication (RSSI) profiles, of a Zigbeebased sensor network inside a car. The results in [4-6] provide comprehensive intra-car wireless channel statistics including its power delay profile (PDP), coherence bandwidth and coherence time. Other fading statistics such as level-crossing rate (LCR) and average fading duration (AFD) have also been measured by the cited works above. Ultra-wideband (UWB) technology is also considered for short range communication within a car due to its low power requirements and high data rate. For such systems, $[2,7,8]$ measured channel characteristics such as impulse response and extraction of impulses arriving at the receiver in clusters due to multi-path effects. The cited works are essential for designing and implementing intra-car transmitter and receiver systems (antenna, modulation technique, equalization algorithms, symbol rate and shape, MAC, etc.) with good performance. In contrast to the previous works, we test and evaluate the performance of multi-hop intra-car WSN on the commercial TelosB platform, whose hardware and software have already been established for the $2.4 \mathrm{GHz}$ ISM band.

\section{B. Collection Tree Protocol}

The Collection Tree Protocol (CTP) is a multi-hop collection protocol, designed to route data from every node on a network to one or more self-declared root nodes, based on minimum cost trees. In [3], where CTP Noe is introduced, the routing gradient metric used is the expected number of transmissions, denoted by ETX. The calculation of the ETX value for each node is done as follows: consider two nodes $a$ and $b$ such that node $a$ is the parent, then we have:

$$
\begin{gathered}
\operatorname{ETX}_{b}=\operatorname{ETX}_{a}+\text { ETX of link } b \rightarrow a ; \\
\operatorname{ETX}_{\text {Root }}=0
\end{gathered}
$$

where the ETX of a link is estimated by a link estimator in a distributed fashion [9]. Given a choice of valid routes, CTP simply chooses the one with the lowest ETX towards the root; note that this routing mechanism also allows for on-line data path validation, since the ETX values should decrease on a packet's path to a receiver. In addition, topology information is updated via broadcasted beacon frames with adaptive time intervals: if the topology is stable and no new nodes are added or removed, the beaconing rate is reduced; on the other hand, if the topology is constantly changing then nodes will have fast beaconing in an attempt to distribute the most up-to-date routing information. For intra-vehicle wireless networks, adaptive beaconing appears to have a reasonable overhead, as experimental results in [6] show that the coherence times are large; in other words, the intra-vehicle slow-fading channels yield a stable topology, and beacon rate tends to decrease under static conditions.

\section{EXPERIMENTAL SETUP}

\section{A. Sensor nodes location and hardware}

For the preliminary tests, we set up a network consisting of four sensor nodes periodically sending data to a single root. The four sensors are attached to the suspension system of a vehicle, and the root is placed inside the instrumental panel close to the driver's seat. The root is connected to a laptop through a USB port for the purposes of logging messages and statistics.

Our sensor nodes are TelosB (TPR2420CA) [10] with data rate $250 \mathrm{kbps}$, RF power $-24 \mathrm{dBm}$ to $0 \mathrm{dBm}$, and receive sensitivity $-94 \mathrm{dBm}$ (typ) through $-90 \mathrm{dBm}(\mathrm{min})$. The sensor nodes' firmware is based on TinyOS 2.x, which is an open source operating system designed for sensor networks. The radio chip of sensor nodes (CC2420) is configured to use Carrier Sense Multiple Access (CSMA) MAC protocol for transmission through the shared wireless medium. 


\section{B. Experimental methodology}

In our experiments we utilize an activator node to send an initial broadcast signal that activates each sensor node and establishes some rudimentary time synchronization; this broadcast signal is manually confirmed to be received by all sensors. The use of a central activation broadcast enables us to update the nodes' configurations by reprogramming only the activator mote. As such, the activator mote can use this broadcast signal to dictate the transmit intervals, transmission powers, and radio channel for nodes. It can also be used to stop and restart an experiment. After the activation step, each sensor node starts to periodically transmit the packets towards the collection root connected to a laptop through a USB port for the purposes of logging messages and statistics. Finally, the log messages are collected for the post-experiment analysis, where we obtain performance metrics and the network topology. This methodology provides an overall framework for experimentation under different network parameters (and collection protocols).

\section{Scenarios}

Our experiments are performed under several vehicular scenarios. The test car is parked in a covered area parking, where there is little foot traffic and some other cars are parked nearby; however, depending on the test time, they may be adjacent to the test vehicle or not. We run the experiment under static conditions, which implies that the engine is turned off, there is no passenger sitting inside the car and WiFi interference is negligible. We then run the experiments under dynamic in-vehicle conditions; namely, engine-on, passengers-move-in-and-out (two persons change their status with a constant time interval), and with WiFi interference. At each run of experiment, one of the aforementioned dynamic condition is exerted to the testbed.

\section{Parameters}

The experiment parameters for the data collection system include packet generation rate, transmission power and packet size. The packet generation rate is defined to be the number of packets that a node generates per second, which is configured to be either 10 or $15 \mathrm{pkts} / \mathrm{sec}$. The transmission powers of nodes take the values $-20 \mathrm{dBm}$, $-10 \mathrm{dBm}$, or $0 \mathrm{dBm}$; the transmission power of the activator node is configured to the highest value $0 \mathrm{dBm}$ for all experiments. The packets size is fixed to 32 bytes including 8 bytes data packet header, 12 bytes payload and 12 bytes CC2420 header.

\section{E. Performance metrics}

Suppose we run an experiment and sensor nodes generate a total number of $M$ packets during the experiment. After the experiment ends, the root successfully receives $N$ packets from the sensor nodes; among the total $N$ received packets, $N_{u}\left(N_{u} \leq N\right)$ packets are unique (distinct) i.e., all duplicates are removed from the count. Delivery rate is then defined to be the fraction of uniquely received packets at the root over the total generated packets in the network $\left(\frac{N_{u}}{M} \times 100 \%\right)$. For a delivered packet, its delivery delay is the time interval between the generation of the packet and its reception at the root. Tx count is defined to be the average number of total transmission attempts from the source to the root (including relay nodes) per received packet at the root.

\section{CTP PERformance Vs. Star Protocol}

In this section, we experimentally quantify the performance gains of multi-hop (CTP) versus single-hop networking (a star protocol). To this end, we first run the experiments of CTP and the star protocol under static and dynamic conditions (passengers-move-in-and-out). We present the results of reliability of both protocols under such scenarios and then provide a discussion on communication and delay trade-offs. Next, we increase the number of sensor nodes to eight and then test the reliability of both protocols for the resultant larger network. 


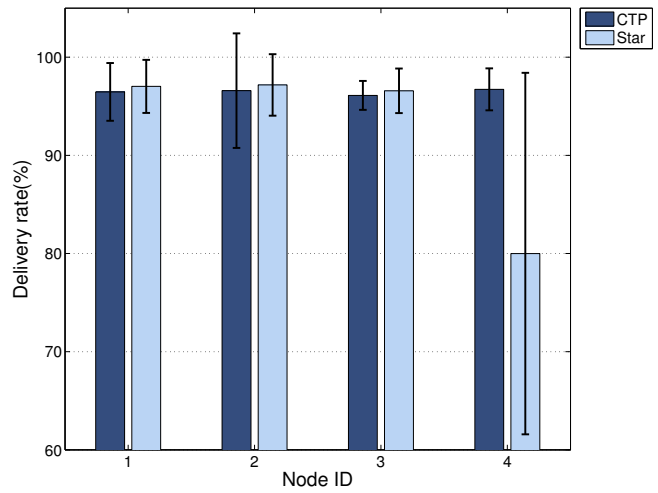

(a) Static condition

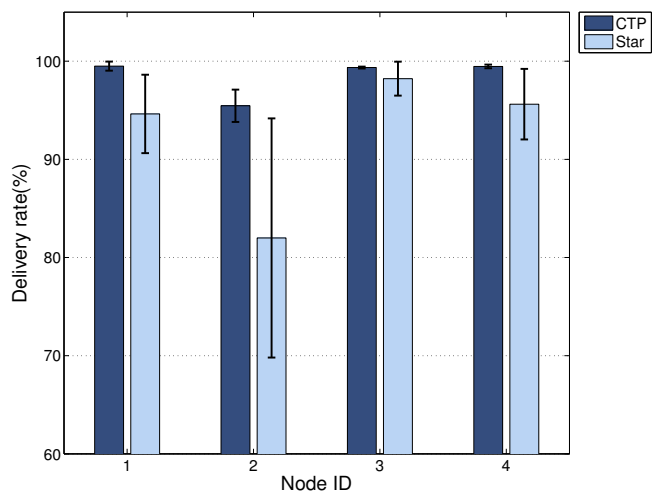

(b) Passengers-move-in-and-out scenario

Fig. 1. CTP and the star protocol delivery rate. (a) Static conditions, Tx power $-20 \mathrm{dBm}$ and packet generation rate 15 pkts/sec per node (b) Passengers-move-in-and-out scenario, Tx power $-10 \mathrm{dBm}$ and packet generation rate $10 \mathrm{pkts} / \mathrm{sec}$ per node. The error bars show one standard deviation from the mean over 5 six-minutes experiments.

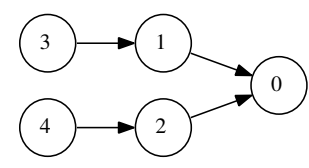

(a) Static condition

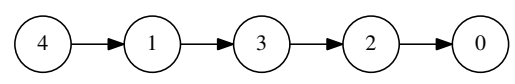

(b) Passengers-move-in-and-out scenario

Fig. 2. Multi-hop CTP topology under (a) static conditions (b) passengers-move-in-and-out scenario.

\section{A. Reliability}

In a star protocol all nodes transmit to the root directly; therefore, if some node-to-root links experience deep channel fading then the quality of service for that node and overall system performance degrade. To implement a star protocol, we force sensor nodes to send beacon messages only to the root; thus, all nodes always choose the root as the next hop and yield a single-hop star topology. In the first experiment, CTP is tested versus the star protocol under static conditions. The results shown in Fig.1(a) demonstrate that CTP provides high delivery rate across all nodes, while the performance variance of different nodes with the star protocol is significant e.g., node 4 achieves about $78 \%$ average delivery rate with a relatively large confidence interval.

Additionally, the delivery rate under a passengers-move-in-and-out scenario is shown in Fig.1(b), which illustrates that CTP outperforms the star protocol under dynamic conditions as well. Indeed, star protocol performance deteriorates under dynamic conditions, while CTP sustains its high delivery rate. The multi-hop topology of CTP under these scenarios is shown in Fig. 2. Our preliminary results indicate that CTP outperforms the star protocol from the reliability and robustness viewpoint under static and dynamic conditions (with passengers in this case).

\section{B. Communication and latency trade-offs}

Reliability is usually desirable for wireless sensor networks, however other metrics like the number of transmissions per packet and latency may also be of interest. While comparing multi-hop with single-hop, some trade-offs emerge. Specifically, multi-hop topology may require more transmissions per each generated packet due to relay nodes, but nodes can transmit with lower power to achieve the same delivery rate as single-hop. Moreover, singlehop latency is smaller than that of multi-hop. But, a single-hop topology is not always reliable and retransmissions (due to CRC errors) are required, which increase latency and power consumption. Table I shows Tx count and delay performance for received packets under static and passengers-move-in-and-out scenarios, which confirms that a star protocol requires fewer transmissions and incurs lower packet delay. However, the delivery rate of CTP is higher than that of a star protocol. Our other experiments show that CTP can achieve the same reliability as the star protocol with $1 / 10^{t h}$ Tx power, but it requires up to $69 \%$ higher Tx count per each generated packet. 


\begin{tabular}{cccccc}
\hline Condition & Tx power & Protocol & Tx count & Delay & Delivery rate \\
\hline \multirow{2}{*}{ Static } & \multirow{2}{*}{$-20 \mathrm{dBm}$} & Star & 1.14 & 14.36 & $92.69 \%$ \\
& & CTP & 1.72 & 37.35 & $96.43 \%$ \\
\hline \multirow{2}{*}{ Passengers } & \multirow{2}{*}{$-10 \mathrm{dBm}$} & Star & 1.22 & 16.72 & $92.59 \%$ \\
& & CTP & 2.54 & 82.92 & $98.41 \%$ \\
\hline
\end{tabular}

TABLE I

CTP AND THE STAR PROTOCOL PERFORMANCE. TX COUNT AND DELAY ARE CALCULATED BASED ON THE RECEIVED PACKETS.

\section{Larger network}

As another experiment setup, the test network is expanded to eight clients. Fig. 3 graphically illustrates the nodes

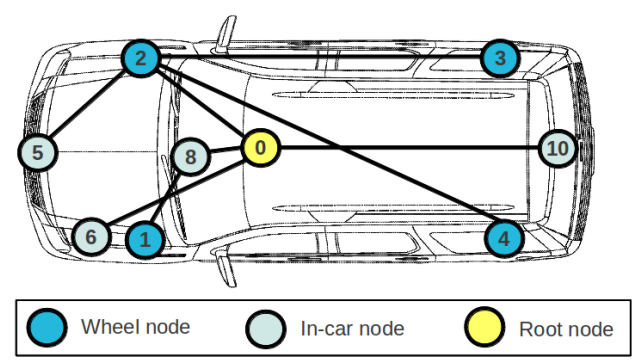

Fig. 3. The multi-hop topology of CTP under static conditions, Tx power $-10 \mathrm{dBm}$ and generation rate 5 pkts/sec per node

placement around the car, where the root (node 0) is located inside the instrumental panel, close to the driver's seat; nodes $1,2,3$ and 4 are mounted on the suspension system; node 8 is located on the dashboard, next to the light sensor; and node 10 is placed in the middle of the trunk. The packet generation rate is 5 pkts/sec per node and the transmission power is $-10 \mathrm{dBm}$. CTP collection routes are shown in Fig.3. The delivery rates of both protocols are shown in Fig. 4, which illustrates that all nodes in CTP achieve higher delivery rate than the star protocol. In fact, the different nodes in the star protocol achieve variant levels of reliability, but CTP will reduce variance in performance among the different nodes and all of them achieve higher than $98 \%$ delivery rate. This experimental result verifies that CTP can outperform single-hop communication from the reliability viewpoint. 


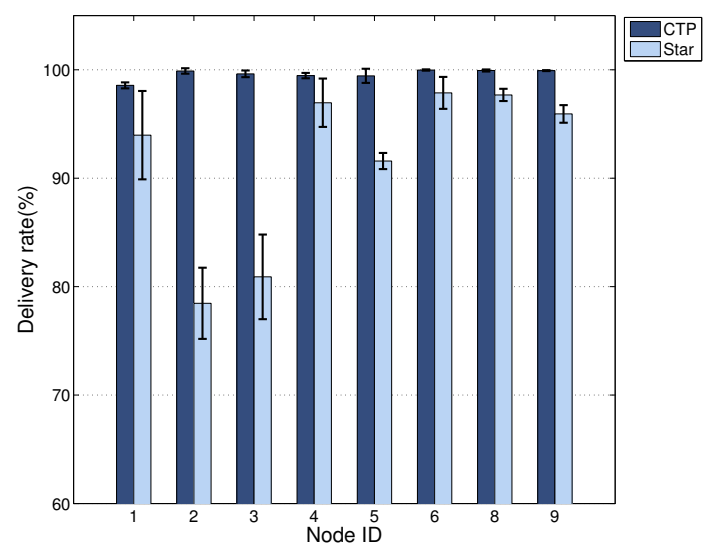

Fig. 4. CTP delivery rate vs. the star protocol under static conditions for 8-nodes network. CTP achieves reliable performance across all nodes.

\section{Adaptive Performance of CTP}

We run CTP experiments under dynamic conditions such as when the engine is on, as the passengers move in and out of the vehicle, or when there exists WiFi interference. Our results illustrate the adaptive behavior of CTP under such conditions.

\section{A. Engine-on scenario}

As the first dynamic case, we test CTP performance under the engine-on scenario with the packet generation rate $10 \mathrm{pkts} / \mathrm{sec}$ per node and the transmit power either $-10 \mathrm{dBm}$ or $0 \mathrm{dBm}$. According to Table II, turning the

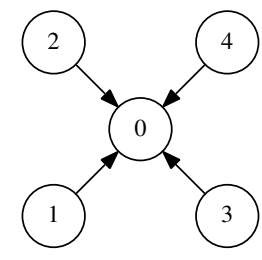

(a) Star topology

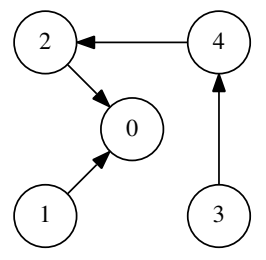

(b) Multi-hop topology

Fig. 5. CTP topology under the engine-on scenario. Fig. (a) shows the topology at the beginning of experiment, which switches to Fig. (b) after a while.

engine on does not have considerable effects (compared with the static conditions) on network performance for both power levels. However, CTP achieves its high delivery performance via a multi-hop topology. At the beginning of

\begin{tabular}{ccccc}
\hline Condition & Tx power & Delivery rate(\%) & Tx count & Delay(ms) \\
\hline \multirow{2}{*}{ Static } & $-10 \mathrm{dBm}$ & 99.76 & 1.69 & 27.98 \\
& $0 \mathrm{dBm}$ & 99.75 & 1.23 & 17.51 \\
\hline \multirow{2}{*}{ Engine on } & $-10 \mathrm{dBm}$ & 99.70 & 1.66 & 23.95 \\
& $0 \mathrm{dBm}$ & 99.67 & 1.86 & 28.19 \\
\hline \multirow{2}{*}{ Passengers } & $-10 \mathrm{dBm}$ & 98.41 & 2.54 & 82.92 \\
& $0 \mathrm{dBm}$ & 99.82 & 1.44 & 24.49 \\
\hline
\end{tabular}

TABLE II

CTP PERFORMANCE UNDER DYNAMIC SCENARIOS VS. STATIC CASE 
experiment, the network topology is a single-hop as depicted in Fig. 5(a). After a while, the topology changes to a multi-hop as shown in Fig. 5(b) where nodes 2 and 4 are selected as the next hop by nodes 4 and 3 respectively. This topology change verifies that CTP adaptively chooses the next hop to construct a multi-hop topology.

\section{B. Passengers-move-in-and-out}

In the second dynamic scenario, CTP performance is evaluated while passengers move in and out of the vehicle. Table II shows CTP performance under this condition. In this scenario, we also obtain RSSI traces which are shown in Fig. 6. At the beginning of experiment all four nodes choose the root as the next hop. However, after some time nodes 3 and 4 select node 1 as the next hop for which RSSI values are higher. Therefore, a multi-hop approach provides the opportunity to dynamically adapt to slow changes in link quality.

\section{WiFi interference}

In this section, we present the results of CTP performance under WiFi interference. The sensor nodes are configured to operate on channel 22 under 802.15.4 standard (channel 11 of 802.11 standard), where the local WiFi is mainly used. The packet generation rate is $10 \mathrm{pkts} / \mathrm{sec}$ per node and the transmission power is $0 \mathrm{dBm}$. We first run the experiment while WiFi interference is negligible. Under such conditions, we observe that all nodes transmit within a single hop towards the root. We then increase interference by streaming a video on a close proximity computer; where the topology changes to a line. Fig. 7 shows the topology for both cases with and without
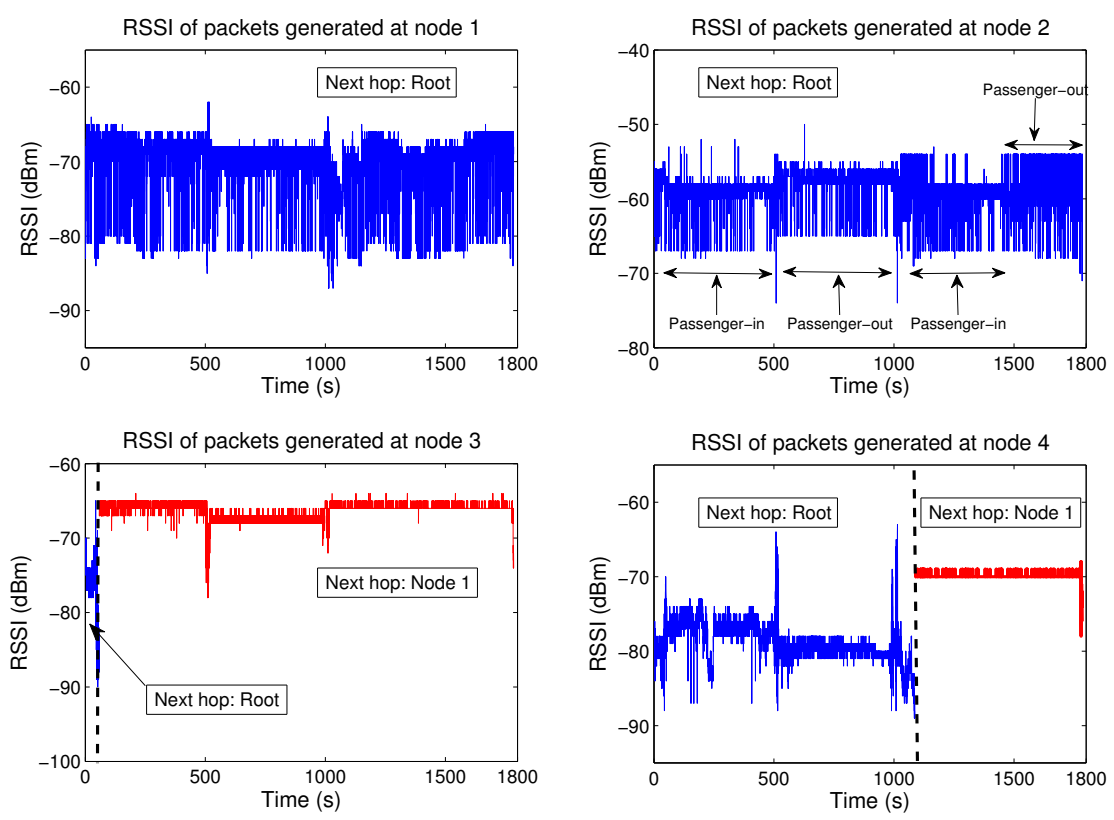

Fig. 6. RSSI traces under passengers-move-in-and-out scenario and Tx power $0 \mathrm{dBm}$. When the link quality of nodes 3 and 4 to the root become bad, they choose node 1 as the next hop. In the graphs, vertical dashed lines indicate topology change points and text-arrows show the time intervals of walking in or out of the vehicle (the same in all sub-figures).

interference. As discussed in Section II, CTP is based on a greedy algorithm to minimize the ETX quantity, which represents the expected number of transmissions. Hence, CTP always chooses opportunistically a path with the lowest ETX value as the next hop. Therefore, the topology transition from star to line under heavy WiFi suggests that a line minimizes the ETX value. However, the minimum may be local and not global. 


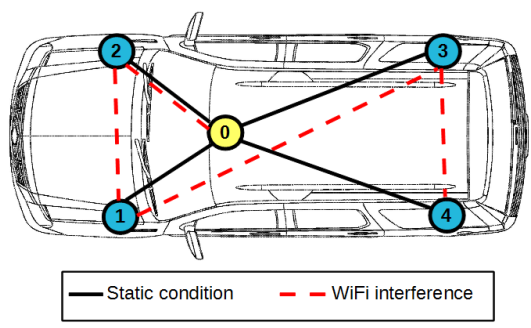

Fig. 7. Network topology with and without WiFi interference. It changes from star to line under heavy WiFi interference.

\section{CONCLUSION}

In this paper, we have evaluated the performance of multi-hop networking for intra-car wireless sensor networks, and showed its superior reliability over a single-hop star protocol. Our results show that the delivery rate of a node in a star protocol can be as bad as $78 \%$, while CTP can achieve a reliability higher than $95 \%$ for all nodes, in most of the scenarios. We also tested CTP under dynamic conditions, such as with the car engine on, with passengers moving in and out of the car, and with WiFi interference. Our experimental results demonstrate that the multi-hop approach enhances certain aspects of the network performance under these scenarios, and such system may be suitable for intra-car environments, based on the robustness of their operation in harsh environments. In future works, we plan to explore hybridized collection protocols that blend reliability with other desired network properties.

\section{REFERENCES}

[1] H.-M. Tsai, O.K. Tonguz, C. Saraydar, T. Talty, M. Ames, and A. Macdonald. Zigbee-based intra-car wireless sensor networks: a case study. Wireless Communications, IEEE, 14(6):67 -77, december 2007.

[2] W. Niu, J. Li, S. Liu, and T. Talty. Intra-vehicle ultra-wideband communication testbed. In Military Communications Conference, 2007. MILCOM 2007. IEEE, pages 1-6. IEEE, 2007.

[3] O. Gnawali, R. Fonseca, K. Jamieson, D. Moss, and P. Levis. Collection tree protocol. In Proceedings of the 7th ACM Conference on Embedded Networked Sensor Systems, SenSys '09, pages 1-14, New York, NY, USA, 2009. ACM.

[4] O.K. Tonguz, H.M. Tsai, T. Talty, A. Macdonald, and C. Saraydar. Rfid technology for intra-car communications: A new paradigm. In Vehicular Technology Conference, VTC Fall. 2006 IEEE 64th, pages 1-6. IEEE, 2006.

[5] H.M. Tsai, W. Viriyasitavat, O.K. Tonguz, C. Saraydar, T. Talty, and A. MacDonald. Feasibility of in-car wireless sensor networks: A statistical evaluation. In Sensor, Mesh and Ad Hoc Communications and Networks, 2007. SECON'07. 4th Annual IEEE Communications Society Conference on, pages 101-111. IEEE, 2007.

[6] A.R. Moghimi, H.-M. Tsai, C.U. Saraydar, and O.K. Tonguz. Characterizing intra-car wireless channels. Vehicular Technology, IEEE Transactions on, 58(9):5299 -5305, nov. 2009.

[7] W. Niu, J. Li, and T. Talty. Intra-vehicle uwb channel measurements and statistical analysis. In Global Telecommunications Conference, 2008. IEEE GLOBECOM 2008. IEEE, pages 1-5. IEEE, 2008.

[8] W. Niu, J. Li, and T. Talty. Intra-vehicle uwb channels in moving and staionary scenarios. In Military Communications Conference, 2009. MILCOM 2009. IEEE, pages 1-6. IEEE, 2009.

[9] R. Fonseca, O. Gnawali, K. Jamieson, S. Kim, P. Levis, and A. Woo. The collection tree protocol (TEP 123). http://www.tinyos.net/tinyos-2.x/doc/html/tep123.html, 2006.

[10] MEMSIC. Telosb specification. http://www.memsic.com. 